\title{
Optically induced multi-particle structures: multi-dimensional energy landscapes
}

\author{
Luciana C. Dávila Romero, Justo Rodríguez and David L. Andrews \\ Nanostructures and Photomolecular Systems, School of Chemical Sciences, University of East \\ Anglia, Norwich NR4 7TJ, United Kingdom
}

\begin{abstract}
Recent quantum electrodynamical studies on optically induced inter-particle potential energy surfaces have revealed unexpected features of considerable intricacy. The exploitation of these features presents a host of opportunities for the optical fabrication of nanoscale structures, based on the fine control of a variety of attractive and repulsive forces, and the torques that operate on particle pairs. Here we report an extension of these studies, exploring the first detailed potential energy surfaces for a system of three particles irradiated by a polarized laser beam. Such a system is the key prototype for developing generic models of multi-particle complexity. The analysis identifies and characterizes potential points of stability, as well as forces and torques that particles experience as a consequence of the electromagnetic fields, generated by optical perturbations. Promising results are exhibited for the optical fabrication of assemblies of molecules, nanoparticles, microparticles, and colloidal multi-particle arrays. The comprehension of mechanism that is emerging should help determine the fine principles of multi-particle optical assembly.
\end{abstract}

Keywords: Optical binding, optical manipulation, potential energy landscapes, quantum electrodynamics, selfassembly, nanophotonics, nanofabrication, nanoparticles

\section{INTRODUCTION}

Recently, there has been growing interest in experimental studies of a new form of interaction between neutral particles, observed in the presence of laser light. Most readily observable in micron and sub-micron sized particles, and often producing sizeable forces of attraction, the phenomenon has become known as 'optical binding', a term first introduced by one of its pioneers, Jean-Marc Fournier. ${ }^{1}$ Commonly, such effects are studied in optical traps which afford a relatively static environment, and which thereby facilitate resolution and measurement of the optically induced forces. ${ }^{2,3}$ Moreover, the radiation used for the trapping is itself engaged in producing the optical binding effect. As the fundamental electrodynamic mechanisms of the optically induced interaction process have become better understood, ${ }^{4-6}$ it has become apparent that the original descriptor 'binding' is unduly limiting, and in some cases potentially misleading. In general, the effect of the light is to confer on particles of matter an intricately patterned potential energy landscape, in which the presence of numerous local maxima and minima signify possibilities for both attractive and repulsive forces, as well as conferring significant torques. ${ }^{7-9}$ The exploitation of such features presents a host of opportunities for the optical fabrication of nanoscale structures.

In our recent work on pair interactions we identified the possibility of creating variously ordered and patterned arrays, based on optically induced forces. In this paper we report the first results to emerge from the next stage in extending these studies to a more detailed characterization of such multi-particle structures. Here, we explore the first detailed potential energy surfaces for a group of three particles irradiated by a polarized laser beam. It emerges that this three-particle system introduces novel features that do not arise in simple pair interactions, signifying the onset of more complex 'optical matter' behavior. As such, the study should pave the way for developing more advanced, generic models of multi-particle complexity. The present analysis can already identify and characterize potential points of stability, as well as forces and torques generated by the applied optical fields. The results appear to offer considerable promise for the optical fabrication of particle assemblies, and a better understanding of how to control the responsible mechanisms. 


\section{OPTICALLY INDUCED PAIR POTENTIAL ENERGY: QED}

Studies based upon quantum electrodynamics reveal the detailed mechanisms by means of which light can significantly modify inter-particle coupling forces. ${ }^{6}$ Fundamentally, the coupling involves four photon-particle coupling events - the absorption of a real (laser) photon at one location and stimulated emission of an identical photon at another location, mediated by the annihilation and creation of a virtual photon that propagates across the intervening space. In an explicitly classical description the interacting particles, which are generally in their ground electronic states, are considered to generate short-lived induced moments (through their interaction with the radiation), the coupling of which produces an accompanying force.

For the quantum electrodynamic analysis of this system it is appropriate to use the Power-Zienau-Woolley approach, ${ }^{10}$ in which the Hamiltonian representing each fundamental interaction between the electromagnetic field and a particle $\xi$, in the electric-dipole approximation, is as follows; ${ }^{6}$

$$
H_{\mathrm{int}}^{\xi}=-i \varepsilon_{o}^{-1} \sum_{\mathbf{k}, \lambda}\left(\frac{\hbar c k \varepsilon_{o}}{2 V}\right)^{1 / 2} \sum_{\xi} \boldsymbol{\mu}(\xi) \cdot\left[\mathbf{e}^{(\lambda)}(\mathbf{k}) a^{(\lambda)}(\mathbf{k}) \exp \left(i \mathbf{k} \cdot \mathbf{R}_{\xi}\right)-\overline{\mathbf{e}}^{(\lambda)}(\mathbf{k}) a^{\dagger(\lambda)}(\mathbf{k}) \exp \left(-i \mathbf{k} \cdot \mathbf{R}_{\xi}\right)\right]
$$

where $\boldsymbol{\mu}(\xi)$ denotes the electric-dipole operator; $\mathbf{R}_{\xi}$ is the position vector of dielectric particle $\xi ; V$ is the quantization volume; $a$ and $a^{\dagger}$ are photon annihilation and creation operators, respectively; e represents the polarization unit vector, with $\overline{\mathbf{e}}$ being its complex conjugate. Summation is taken over modes indexed by wave-vector $\mathbf{k}$ and polarization label $\lambda$. The inter-particle interaction potential, evaluated through fourth-order perturbation theory, for the case of laser photon absorption at a particle $A$ and stimulated emission at a particle $B$ is as follows;

$$
\Delta E^{A B}(k, \mathbf{R})=\left(\frac{n \hbar c k}{\varepsilon_{o} V}\right) \operatorname{Re}\left[e_{i}^{(\lambda)} \alpha_{i j}^{A}(k) V_{j k}^{ \pm}(k, \mathbf{R}) \alpha_{k l}^{B}(k) \bar{e}_{l}^{(\lambda)} \exp (-i \mathbf{k} \cdot \mathbf{R})\right]
$$

Here $\mathbf{R}=\mathbf{R}_{A}-\mathbf{R}_{B} ; n$ is the number of laser photons within a quantization volume $V$, given by the irradiance of the laser beam, $I=n \hbar c^{2} k / V$, and we have introduced the dynamic polarizability tensor $\alpha_{i j}^{\xi}(-k, k)=\alpha_{i j}^{\xi}(k)$ of a particle $\xi^{11}$; lastly, the fully retarded resonance dipole-dipole interaction tensor features in its general form ${ }^{12}$;

$$
V_{j k}^{ \pm}(k, \mathbf{R})=\frac{\exp (\mp i k R)}{4 \pi \varepsilon_{o} R^{3}}\left\{(1 \pm i k R)\left(\delta_{j k}-3 \hat{R}_{j} \hat{R}_{k}\right)-(k R)^{2}\left(\delta_{j k}-\hat{R}_{j} \hat{R}_{k}\right)\right\}
$$

An analogous mechanism for the particle-photon events of absorption at position $B$ and stimulated emission at position $A$ needs to be taken into account for the calculation of the total energy shift responsible for optical binding. ${ }^{6}$

For microparticles whose electronic properties are neither those of a single large molecule, nor exactly those of a molecular aggregate, the electrical response properties that appear in the equations should be translated into bulk quantities; the polarizability becomes the linear susceptibility, for example. At this level, confinement effects associated with specifically nanoscale particles can also be accommodated in the theory. In view of the usual trapping environment, account can also be taken of the optical properties of the medium supporting the particles. In most experiments it has been shown that the relative values of the refractive index between the particles and the surrounding medium significantly influence the optical binding phenomena, modifying the positions of stability. With the incorporation of Lorentz field factors, the dependence on $k R$ changes to a dependence on $n(c k) k R$, where the multiplier is the complex refractive index. 


\section{OPTICALLY INDUCED ENERGY LANDSCAPES}

Previous studies of the optically induced inter-particle potential energy landscapes for spherical particles in a single plane wave have revealed intricate features conductive to the production of a variety of attractive and repulsive forces, and torques, engendering the formation of several multi-particle structures. ${ }^{7-9}$ Here, we use a different parameterization for the potential energy that is later shown to reveal additional features on this potential. The Cartesian coordinate system for two particles and a plane wave geometric configuration is defined as shown in Figure 1; the Poynting vector is along the $y$ axis and the polarization along the $z$ axis. In this condition, applying equation (1.2) to spherical particles, the total energy shift can be written as;

$$
\Delta E=\left(\frac{2 I \alpha^{2} k^{3}}{\varepsilon_{o} c(k R)^{3}}\right)\left[(\cos (k R)+k R \sin (k R))\left(1-3 \hat{R}_{\mathrm{z}}^{2}\right)-(k R)^{2} \cos (k R)\left(1-\hat{R}_{\mathrm{z}}^{2}\right)\right] \cos \left(k R_{\mathrm{y}}\right)
$$

where $R$ is the particle separation, $\hat{R}_{\mathrm{z}}$ is the projection on the $z$ axis of an unit vector on the direction of the interparticle displacement, and $R_{\mathrm{y}}$ is the corresponding projection of the full vector on the y axis. The energy shift is then expressed as a function of the Cartesian components of the displacement vectors $R_{x}, R_{y}$ and $R_{z}$, and is an even function of these parameters.



Figure 1. Two particles in a plane wave of polarization $\mathbf{e}$, and wave-vector $\mathbf{k}$. The vector $\mathbf{R}$ denotes the displacement between the particles $A$ and $B$, where $A$ can be considered as located at the origin. The $\mathrm{z}$ and y axes are defined along $\mathbf{e}$ and $\mathbf{k}$, respectively.

\section{DISCUSSION}

In most applications it is possible to regard the laser irradiance as being essentially time-invariant. Generally, even if pulsed radiation is used in an experiment the pulse repetition interval is in fact sufficiently short, compared to the particle diffusion times, that the input operates as being effectively constant in time. In such an environment, particles are individually displaced by gradient forces to the neighboring maxima of irradiance in the beam structure known as optical traps - the latter being identifiable with the beam axis in the simplest Gaussian profile case. The location of any such optical trap signifies a relatively static environment, one that facilitates the resolution and measurement of any optically induced pair forces. A simplifying factor is that these traps are generally much larger in dimension than the typical particle separation, and therefore the applied irradiance may also be taken to be spatially constant, making the optically induced inter-particle potential entirely separable from the gradient forces. It can also be assumed that the 
separation of the particles is sufficiently large - and the laser pulses intense enough - to justify neglect of the CasimirPolder potential with respect to its laser-induced counterpart; this assumption is also expected to apply in most cases, when particle separations are of the order of the laser wavelength. Under this combination of conditions, the optically induced inter-particle potential operates to translate the particles towards separations that correspond to its energy minima.

An analysis of the spatial dependence of the optical binding forces reveals the arrangement of particles in an optical trap. Assuming spherical particles, landscapes of the potential energy surfaces generated by the optically induced pair forces are shown as a function of the Cartesian components of the separation vectors, $R_{x}, R_{y}$ and $R_{z}$, in Figure 2 . The distance scales on this and subsequent graphs are normalized by plotting results against dimensionless variables scaled by $k$ (whose value is $2 \pi$ times the inverse wavelength of the applied radiation). One of the interesting features that is immediately evident, in Figure 2 (a), is the sequence of minima for particles on the $y$ axis that are responsible for longitudinal optical binding, which quickly become of higher energy with increasing $R_{x}$, Figure 2 (b). Other minima may also be observed in Figure 2 (a); however, they can be seen to become more energetically favorable by increasing $R_{x}$, as is apparent from Figure 2 (b), (c), and (d), suggesting that they are not three-dimensional minima.

A detailed exploration of the dependence of these two-dimensional energy minima on $R_{x}$, and on others not shown in the ranges chosen for the figure, reveals that their potential energy always decreases as a function of $R_{x}$, accompanied by a contraction in positioning of the two dimensional minima on $R_{z}$, reaching a final point of stability when $R_{z}=0$. An example can be shown for the minimum in the position $\left(k R_{y}, k R_{z}\right)=(3.0,9.5)$ when $k R_{x}$ is assumed to be fixed at 0 , in Figure 2 (a); on increasing $k R_{x}$ to 4 and 6 , the stability points move to the new positions $(3.0,8.0$ ), and $(3.0,6.5)$, respectively, with the value of the energy minimum always decreasing; finally the system finds a point of minimum energy on $k R_{x}$, when its value is 8.6 , at $\left(k R_{y}, k R_{z}\right)=(3.0,0.0)$. In other words, particles in the $y z$ plane are effectively tunneled through potential energy channels to three-dimensional minima of potential energy on the $x y$ plane. Thus, the final positioning of the pair is the always with its mutual displacement vector aligned transverse to the polarization of the plane wave. The potential in this plane, illustrated in figure 3 , is a prototypical template for the optical fabrication of multi-particle structures.

It is apparent that these principles afford a tractable basis for extension to a system of $N$ spherical particles, on irradiation with a linearly polarized plane wave. In general, determination of the optically induced potential that is experienced by the whole assembly requires the evaluation of equation (2.1) for $N ! /(N-2)$ ! particle pairs - obviously making analysis of the system significantly more complex for large number of particles. It is interesting and expedient to approach the problem by considering the possibility of optically fabricating structures for the simplest case of three particles - a system that represents the key prototype for developing generic models of multi-particle complexity. In this case, evaluating the total optically conferred energy of the system requires the consideration of three pair interaction potentials, and the particle structures that can be formed are derivable from the template shown in figure 3 . It is evident that the most energetically favorable configuration for a three-particle system is a linear arrangement aligned along the propagation direction, with the particles separated by $k R \cong 3$, followed in order of stability by a collinear arrangement of three particles transverse to both the polarization and propagation directions, and separated by $k R \cong 6$. Less stable three-particle assemblies are collinear arrangements on the $y$ or $z$ axes where one or two particle separations are $k R \cong 6$ or $k R \cong 12$, respectively. Nonetheless other, less simple, structures are also possible; for instance, three particles in the positions $\left(k R_{x}, k R_{y}, k R_{z}\right)=(0,0,0),(0,6,0)$ and $(0,3,8.6)$. The landscapes in figure 2 and 3 also reveal a complex pattern of attractive and repulsive forces along the three axes of the system - features that may become increasingly more complicated when several particles are present in the system. This handful of examples of possible arrangements is indicative of the extensive variety of possible assemblies, and the complexity that can be anticipated with larger number of particles. 
A more detailed exploration of the multi-particle structures and forces requires an analysis of the total potential energy of the system as a function of $3(N-1)$ degrees of freedom for $N$ trapped particles. There is further challenge in identifying and calculating contributions associated with multi-particle processes involving the entangled near-field interactions of more than two particles. Moreover, since the fundamental stimulated scattering process releases throughput radiation essentially unchanged, multiple processes of stimulated scattering have also to be entertained in order to fully understand the formation of stable particle arrays. The full incorporation of such features in a comprehensive analysis represents our objective, and will be subject of future work.
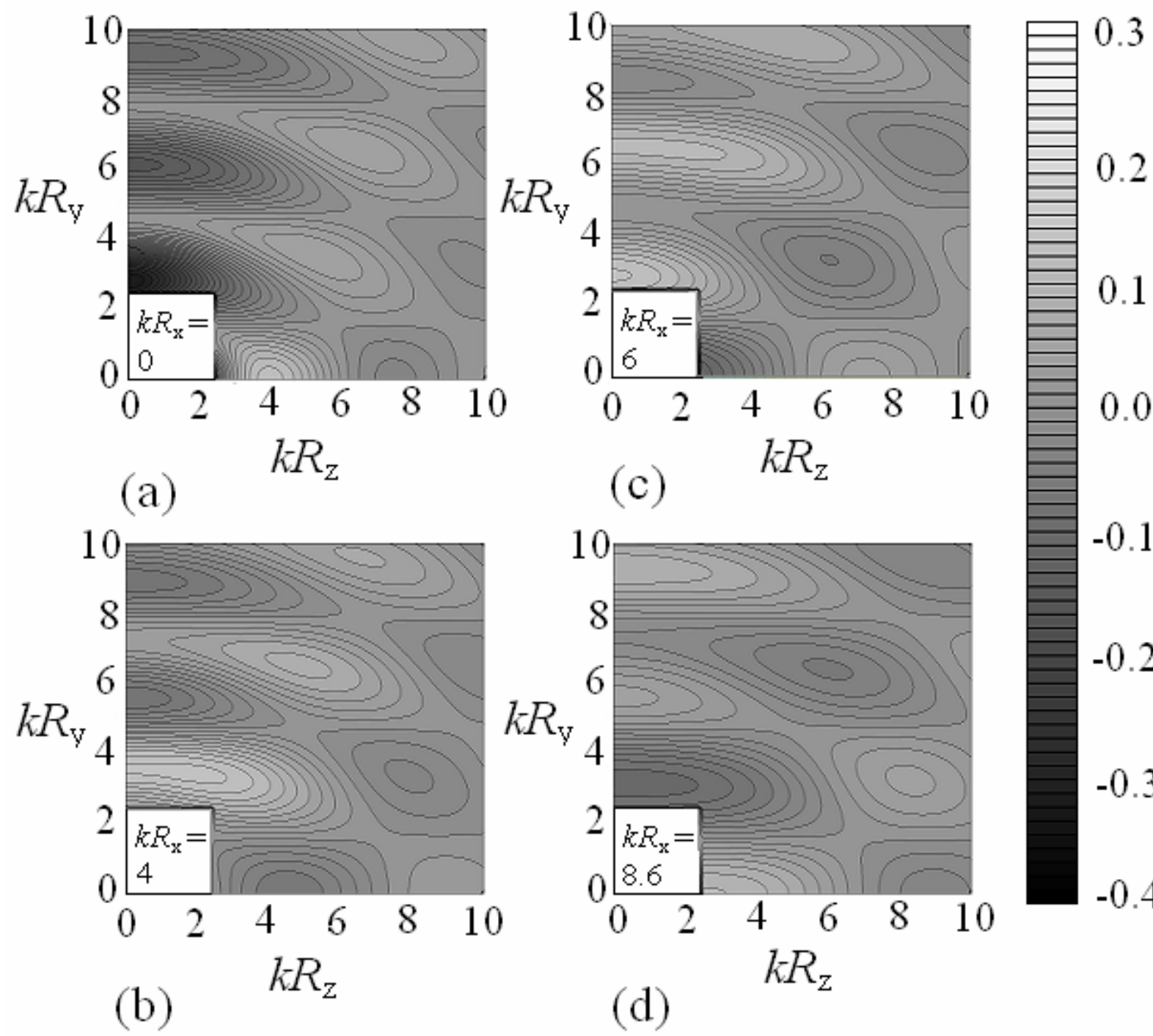

$-0.1$

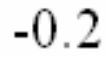

$-0.3$

$-0.4$

Figure 2. Optically induced inter-particle energy surfaces as a function of the particle separation vector, $\mathbf{R}=\left(R_{x}, R_{y}, R_{z}\right)$, for different values of $k R_{\mathrm{x} .}$. The color scale is in $2 I \alpha^{2} k^{3} /\left(\varepsilon_{o} c\right)$ units. 


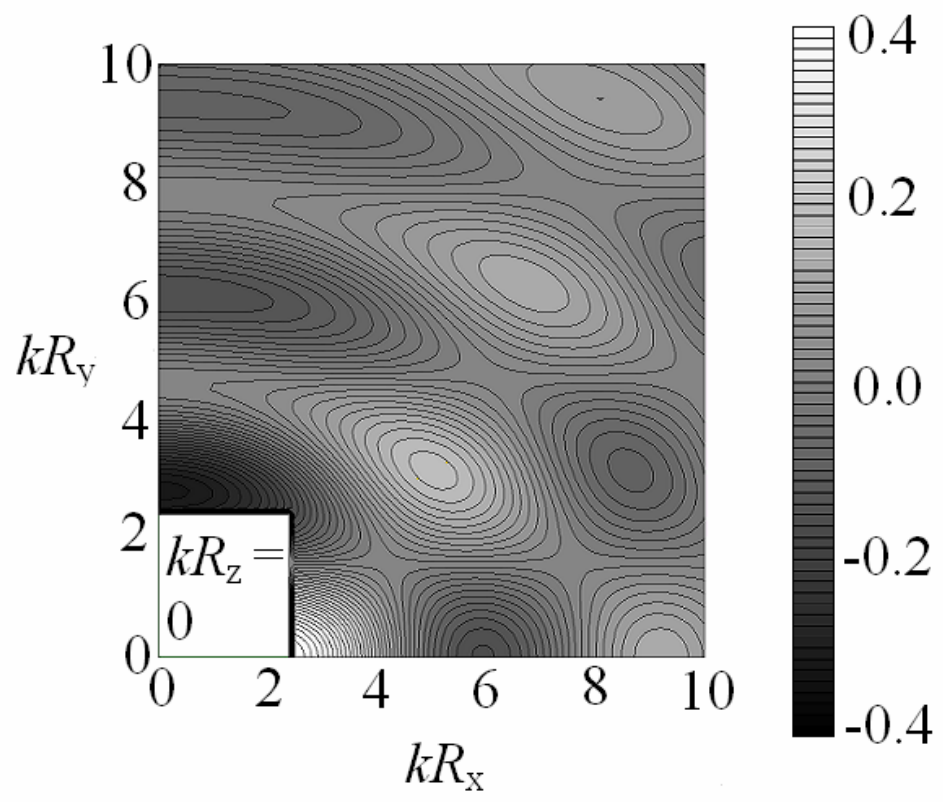

Figure 3. Optically induced interparticle energy surface on the xy plane of figure 1 . The colour scale is in $2 I \alpha^{2} k^{3} /\left(\varepsilon_{o} c\right)$ units.

\section{ACKNOWLEDGEMENTS}

This work is funded by the Engineering and Physical Sciences Research Council

\section{REFERENCES}

1. M. M. Burns, J.M. Fournier, and J.A. Golovchenko, “Optical binding,” Phys. Rev. Lett. 63, $1233,1989$.

2. N. K. Metzger, R. F. Marchington, M. Mazilu, R. L. Smith, K. Dholakia, and E. M. Wright, "Measurement of the restoring forces acting on two optically bound particles from normal mode correlations," Phys. Rev. Lett. 98, $068102,2007$.

3. M. Guillon, O. Moine, and B. Stout, "Longitudinal optical binding of high optical contrast microdroplets in air," Phys. Rev. Lett. 96, 143902, 2007.

4. S. K. Mohanty, J. T. Andrews, and P. K. Gupta, “Optical binding between dielectric particles," Opt. Express 12, 2746, 2004.

5. M. Nieto-Vesperinas, P. C. Chaumet, and A. Rahmani, "Near-field photonic forces." Phil. Trans. R. Soc. Lond, A 362, 719, 2004.

6. D. S. Bradshaw and D. L. Andrews, "Optically induced forces and torques: Interactions between nanoparticles in a laser beam,” Phys. Rev. A 72, 033816, 2005.

7. J. Rodríguez, L. C. Dávila Romero and D. L. Andrews, “Optically induced potential energy landscapes", $J$. Nanophotonics 1, 019503, 2007.

8. L. C. Dávila Romero, J. Rodríguez and D. L. Andrews, "Electrodynamic mechanism and array stability in optical binding”, Opt. Commun. 281, 865, 2007.

9. J. Rodríguez, L. C. Dávila Romero, and D. L. Andrews, “Optical binding: potential energy landscapes and QED” in Complex Light and Optical Forces II, ed. D. L. Andrews, E. J. Galvez, G. Nienhuis, Proc. SPIE, 6905, 69050H, 2008.

10. D. P. Craig and T. Thirunamachandran, Molecular Quantum Electrodynamics, Dover, Mineola, New York, 1998.

11. D. L. Andrews, P. Allcock, Optical Harmonics in Molecular Systems, Wiley-VCH, New York, 2002.

12. R. D. Jenkins, G. J. Daniels, and D. L. Andrews, "Quantum pathways for resonance energy transfer", J. Chem. Phys, 120, 11442, 2004. 